\title{
Relationship between defect density and charge carrier transport in amorphous and microcrystalline silicon
}

\author{
Oleksandr Astakhov \\ Forschungszentrum Jülich, Institute of Energy Research-Photovoltaic, 52425 Jülich, Germany \\ and National Science Center-Kharkov Institute of Physics and Technology, Cyclotron Science and Research Establishment, 61108 \\ Kharkov, Ukraine \\ Reinhard Carius and Friedhelm Finger \\ Forschungszentrum Jülich, Institute of Energy Research-Photovoltaic, 52425 Jülich, Germany \\ Yuri Petrusenko, Valery Borysenko, and Dmytro Barankov \\ National Science Center-Kharkov Institute of Physics and Technology, Cyclotron Science and Research Establishment, 61108 \\ Kharkov, Ukraine \\ (Received 4 July 2008; revised manuscript received 23 December 2008; published 18 March 2009)
}

\begin{abstract}
The influence of dangling-bond defects and the position of the Fermi level on the charge carrier transport properties in undoped and phosphorous doped thin-film silicon with structure compositions all the way from highly crystalline to amorphous is investigated. The dangling-bond density is varied reproducibly over several orders of magnitude by electron bombardment and subsequent annealing. The defects are investigated by electron-spin-resonance and photoconductivity spectroscopies. Comparing intrinsic amorphous and microcrystalline silicon, it is found that the relationship between defect density and photoconductivity is different in both undoped materials, while a similar strong influence of the position of the Fermi level on photoconductivity via the charge carrier lifetime is found in the doped materials. The latter allows a quantitative determination of the value of the transport gap energy in microcrystalline silicon. The photoconductivity in intrinsic microcrystalline silicon is, on one hand, considerably less affected by the bombardment but, on the other hand, does not generally recover with annealing of the defects and is independent from the spin density which itself can be annealed back to the as-deposited level. For amorphous silicon and material prepared close to the crystalline growth regime, the results for nonequilibrium transport fit perfectly to a recombination model based on direct capture into neutral dangling bonds over a wide range of defect densities. For the heterogeneous microcrystalline silicon, this model fails completely. The application of photoconductivity spectroscopy in the constant photocurrent mode (CPM) is explored for the entire structure composition range over a wide variation in defect densities. For amorphous silicon previously reported linear correlation between the spin density and the subgap absorption is confirmed for defect densities below $10^{18} \mathrm{~cm}^{-3}$. Beyond this defect level, a sublinear relation is found i.e., not all spin-detected defects are also visible in the CPM spectra. Finally, the evaluation of CPM spectra in defect-rich microcrystalline silicon shows complete absence of any correlation between spindetected defects and subband gap absorption determined from CPM: a result which casts considerable doubt on the usefulness of this technique for the determination of defect densities in microcrystalline silicon. The result can be related to the inhomogeneous structure of microcrystalline silicon with its consequences on transport and recombination processes.
\end{abstract}

DOI: 10.1103/PhysRevB.79.104205

PACS number(s): 72.20.Jv, 71.23.Cq, 71.55.Jv, 61.80.Fe

\section{INTRODUCTION}

The transport of electronic charge carriers in thin-film silicon (TFS) semiconductors such as amorphous $(a-\mathrm{Si}: \mathrm{H})$ or microcrystalline silicon $(\mu c-\mathrm{Si}: \mathrm{H})$ is strongly influenced by localized defect states arising from structural disorder such as broken bonds [dangling bonds $(\mathrm{db})$ ].

The influence of these dangling-bond defect states in the mobility gap on charge carrier transport in $a$-Si: $\mathrm{H}$ has been studied in great detail [see Ref. 1 and references cited therein for an overview of early work]. The charge state and the density $\left(N_{D}\right)$ of the db states strongly influence the nonequilibrium carrier lifetime and therefore are crucial for any optoelectronic or photovoltaic application. Not to forget that $N_{D}$ will influence the position of the Fermi level and, by carrier trapping, the electric fields in devices such as solar cells. The defect density is strongly affected by the preparation conditions, e.g., when aiming at high deposition rates, and by several external influences such as light illumination or bias stress, which result in a metastable increase of $N_{D} \cdot{ }^{2,3}$ Besides being therefore an important measure of the material quality and optimization parameter for the deposition process of $a-\mathrm{Si}: \mathrm{H}$, the defect density and its relationship to charge carrier dynamics and recombination processes have been of fundamental interest.

For $\mu c-\mathrm{Si}: \mathrm{H}$, which exists in a large variety of structure compositions of crystalline and disordered phase in different amounts and dimensions, structural defects such as dangling bonds are also expected to influence the charge carrier transport, recombination, and device performance. Various reports show such relationships between defect density, position of the Fermi level and defect charge state, carrier lifetime, and 
overall device performance. ${ }^{4-10}$ However, due to the composite structure of $\mu c-\mathrm{Si}: \mathrm{H}$, the situation is considerably more complicated as compared with $a-\mathrm{Si}: \mathrm{H}$. The location of defects, whether being in the amorphous phase, at grain boundaries, or in the crystallites, is not known conclusively. Defects might exist in all phases but are not unambiguously identified so far nor is their individual influence on charge carrier transport understood., ${ }^{5,9-15}$

Between $a-\mathrm{Si}: \mathrm{H}$ and $\mu c-\mathrm{Si}: \mathrm{H}$ - the latter of course already being a class of materials instead of one well-defined material modification - there is an additional form of thinfilm silicon prepared in plasma-enhanced chemical vapor deposition (PECVD) processes with high hydrogen dilution close to the transition to microcrystalline growth. Such material called polymorph, paracrystalline, protocrystalline, or edge material ${ }^{16-20}$ is reported to have reduced defect density, improved stability, and possible medium range order (MRO). ${ }^{21-26}$ Such material is since long used as absorber layer in solar cells, in particular, in the top cells of stacked devices. ${ }^{19,27}$

In the present paper, we will be concerned with all these three types of materials. We investigated the relationship between the defect density and carrier transport. For variation in the defect density, we apply the defect creation by highenergy electron bombardment and subsequent annealing of the defects. ${ }^{15,28-31}$ This approach has been successfully applied to $a-\mathrm{Si}: \mathrm{H}$ and related alloys in the past for reversible variation in the defects over several orders of magnitude. ${ }^{32-40}$ Alternatively also keV electron bombardment for defect production has been reported in the literature. ${ }^{41-45}$ Though the defect creation mechanism is different in the case of $\mathrm{MeV}$ (atom displacement) and $\mathrm{keV}$ (bond breaking), the result measured with electron-spin resonance (ESR) is essentially the same-increase in the dangling-bond density. Both methods have the advantage to access a much wider range of defect densities compared with light-induced degradation. ${ }^{2,46}$ One of the important conclusions from these studies in the past is that the photoconductivity depends on the defect density according to the relation

$$
\sigma_{\mathrm{ph}} \propto N_{S}^{-1},
$$

which can be described by a recombination model for direct capture into neutral defect states. ${ }^{1}$ However, in those earlier studies, such proportionality was only demonstrated over a limited range of defect densities and several experimental results showed considerable deviation from such a behavior. In the present work, we will show the validity of this dependency over almost 3 orders of magnitude of defect densities for all intrinsic amorphous silicon material investigated. In addition, we extend the investigation to $\mu c-\mathrm{Si}: \mathrm{H}$, where we will show that the above relationship is not valid. For microcrystalline silicon, there had been only a few studies where the defect density was controllably varied by electron bombardment and subsequent annealing. ${ }^{47-50} \mathrm{~A}$ systematic investigation of the relationship between defect density and photoconductivity in $\mu c-\mathrm{Si}: \mathrm{H}$ is, to our knowledge, missing so far.

In general, the bombardment-induced dangling-bond defects can be annealed back to the initial state. This therefore allows to study the relationship between defect density, transport, and recombination in one and the same sample. In addition, when applied to doped material, the creation of deep defects results in a shift of the Fermi level, which again can be reversed by annealing. In this case, one can investigate the influence of the Fermi-level position and scan the defects in different energy regions inside the mobility gap. The electron bombardment method has an advantage over alternative approaches, where variation in the defect density is obtained by variation in the deposition process such as deposition temperature or discharge power in glow discharge processes. In this case, it is likely that other material properties, such as hydrogen content, band gap, or structure, in general are affected also and one compares different materials.

In recent years, we have developed an electron bombardment experiment where great care is taken to keep the cold chain between $2 \mathrm{MeV}$ electron irradiation at $100 \mathrm{~K}$, sample transport, storage, handling, and finally measurement. This helped to keep a very high density of generated defects by preventing annealing at ambient. ${ }^{15,28-31}$ While in previous work we were mainly concerned with the effects of electron bombardment on the spin properties in the thin-film silicon materials, in the present report we focus on the interaction between the generated defects and the electronic transport and recombination in the material.

For the investigation of the defects, we use (i) ESR which detects the singly occupied neutral charged db state ${ }^{51}$ and (ii) photoconductivity spectroscopy in the constant photocurrent mode (CPM). ${ }^{52,53}$

The evaluation of the spin density from ESR measurements on thin-film silicon is fairly straight forward. For $a-\mathrm{Si}: \mathrm{H}$ the method is established and the spin density in intrinsic material is considered an appropriate measure for the deep defect density. For undoped $\mu c-\mathrm{Si}: \mathrm{H}$ we have shown recently that also in this material the spin density represents the majority of deep defects. ${ }^{10}$ On the other hand, the use of CPM for the evaluation of the defect density is based on a number of assumptions on the nonequilibrium carrier transport and recombination process in the material and the application has been discussed controversially and studied in great detail. ${ }^{53-55}$ Nevertheless for $a-\mathrm{Si}: \mathrm{H}$, a good correlation is usually found between spin density $\left(N_{S}\right)$ evaluated from ESR measurements and defect density $\left(N_{D}\right)$ determined from CPM spectroscopy. ${ }^{56}$ For $\mu c-\mathrm{Si}: \mathrm{H}$, with its inhomogeneous structure and resulting influence on electronic transport, the use of photoconductivity spectroscopy for the detection of defects has been suggested ${ }^{57-59}$ but in how far in $\mu c-\mathrm{Si}: \mathrm{H}$ a similar relationship between spin density and defect absorption such as in $a$-Si:H exists over a wider range of defect densities has to be shown. This will be another aim of the present paper.

In addition to the measurement of spin density and of CPM spectra, dark conductivity and photoconductivity of the samples were measured before and after irradiation and after each annealing step to monitor the electronic transport and recombination as a function of defect density or Fermi-level position.

We cover the entire range of thin-film silicon materials prepared with a PECVD process at $200{ }^{\circ} \mathrm{C}$ from silane/ 
hydrogen mixtures. The material structure ranges from highly crystalline $\mu c-\mathrm{Si}: \mathrm{H}$ through the transition both on the crystalline and the amorphous growth parameter space to fully amorphous structure.

The results show a number of general similarities in the dependency of electronic transport in $\mu c-\mathrm{Si}: \mathrm{H}$ and $a-\mathrm{Si}: \mathrm{H}$ as a function of defect density and the defect charge changed by a Fermi-level shift, which proves the importance of localized defect states on carrier transport and recombination in all thin-film silicon materials. But there are also distinct differences in the behavior. It will be shown that in $a-\mathrm{Si}: \mathrm{H}$ a recombination model based on direct capture into neutral dangling bonds is valid over 3 orders of magnitude of defect densities. On the other hand for $\mu c-\mathrm{Si}: \mathrm{H}$, the presented results discourage the use of a homogeneous transport and recombination model and shades doubt on the usefulness of photoconductivity-based CPM to measure defect densities in $\mu c-\mathrm{Si}: \mathrm{H}$.

\section{EXPERIMENT}

Samples were deposited with PECVD at $200{ }^{\circ} \mathrm{C}$ from gas mixture of silane and hydrogen. ${ }^{10,60}$ The silane concentration defined by the ratio of the process gas flow [SC $\left.=\mathrm{SiH}_{4} /\left(\mathrm{SiH}_{4}+\mathrm{H}_{2}\right)\right]$ was varied from 3 to $100 \%$ to obtain material with different structure composition. Doping of the samples was achieved through an admixture of 2-150 ppm $\mathrm{PH}_{3}$ with respect to the process gas $\mathrm{SiH}_{4}$. Samples were prepared as films on glass substrate for conductivity measurements and as flakes of pure material encapsulated in quartz tubes for the ESR measurements. The samples were codeposited on Mo foils (Goodfellow MO000250) and roughened borosilicate glass substrates (Corning 7059) of size 4 $\times 15 \mathrm{~mm}^{2}$. Aluminum contacts with $4 \times 0.5 \mathrm{~mm}^{2}$ gap were deposited by electron-beam evaporation for conductivity measurements. Film thicknesses were between 1 and $10 \mu \mathrm{m}$. The film thickness was estimated from the deposition rate and the film mass. For the ESR powder samples, the Mo metal foil was bent after deposition and the peeled-off flakes were weighed and sealed in quartz tubes (Wilmad 710$\mathrm{SQ}-250 \mathrm{M})$ in $500 \mathrm{hPa} \mathrm{He}$ atmosphere.

Conductivity was measured in vacuum in the dark and with illumination from a tungsten halogen lamp with a heat filter (KG3 filter from SCHOTT AG company). The illumination power $\left(\sim 60 \mathrm{~mW} / \mathrm{cm}^{2}\right)$ was set to yield similar photoconductivities in $a-\mathrm{Si}: \mathrm{H}$ such as under AM1.5 illumination. This is a widely used and convenient experimental setup where the optical excitation matches nicely in particular with the optical properties of $a-\mathrm{Si}: \mathrm{H}$. The disadvantage is that the optical excitation spectrum is not well defined and the extraction of the $\eta \mu \tau$ product (quantum-efficiency*mobility*lifetime) of the investigated materials is not possible. This can, however be tolerated in the present case as we are mainly interested in the relative changes in the photoconductivity upon changes in the defect density. Ohmic performance of the metal contacts before and after irradiation was verified. ac mode CPM (Ref. 52) was measured in vacuum using phase-sensitive detection of the signal at the frequency $8.7 \mathrm{~Hz}$. The phase of the CPM signal was used to monitor the possible lifetime variation over the spectrum. The CPM spectra of individual samples before and after irradiation and in different states of annealing were normalized at the onset of saturation of the photoresponse signal. As a relative value for the defect absorption, the relative absorption coefficient was determined at a photon energy of $1.2 \mathrm{eV}$ for $a-\mathrm{Si}: \mathrm{H}$ and $0.9 \mathrm{eV}$ for $\mu c-\mathrm{Si}: \mathrm{H}^{61,62}$ Raman measurements were performed on pieces of the sample on the metal foil with $648 \mathrm{~nm}$ excitation light from an Ar laser. A semiquantitative value for the crystalline volume fraction $I_{C}^{\mathrm{RS}}$ was determined from the Raman spectra as a ratio between intensities of the signals at $520 \mathrm{~cm}^{-1}$ and $500 \mathrm{~cm}^{-1}$ (attributed to the crystalline phase) and the signal at $480 \mathrm{~cm}^{-1}$ (attributed to the disordered phase), i.e., $I_{C}^{\mathrm{RS}}=\left(I_{500}\right.$ $\left.+I_{520}\right) /\left(I_{480}+I_{500}+I_{520}\right)$. $^{63}$

Electron irradiation was performed on films on substrates and powder samples in quartz tubes simultaneously at $100 \mathrm{~K}$ with $2 \mathrm{MeV}$ up to a dose of $1.1 \times 10^{18} \mathrm{~cm}^{-2} \cdot{ }^{28,29}$ Both tubes and substrate samples were handled, transported, and stored in liquid nitrogen $\left(\mathrm{LN}_{2}\right)$. Exposure time to ambient during installation of the samples to the ESR spectrometer or CPM setup was typically 1-3 min. To remove the irradiationinduced $E^{\prime}$ center-an oxygen vacancy in the quartz tubes $^{64}$ —one end of the quartz tube was annealed with a gas burner flame while the other end was kept in a $\mathrm{LN}_{2}$ bath. This procedure completely anneals the $E^{\prime}$ centers while keeping the sample powder at $77 \mathrm{~K}$. The sample can then be measured in the annealed part of the quartz tube. The variation in the spin density after irradiation and annealing was measured on these powder samples. For samples on glass substrate ESR measurements were heavily dominated by the $E^{\prime}$ center generated in the substrate. Therefore, the spin density determined for the powder samples is used for the evaluation of the spin density in coirradiated samples on substrates. Transport measurements were seemingly not affected by the defect generation in the substrate or contacts.

Electron-spin resonance is measured in cw mode in $X$ band in the dark with a Bruker "ELEXSYS" 580 system in a rectangular TM mode cavity. The spin density was calculated after integration of the ESR signal and comparison with a secondary spin standard of sputtered unhydrogenated $a$-Si. ${ }^{65,66}$ This sample is also used for magnetic field calibration.

The samples were stepwise annealed in vacuum $\left(10^{-5} \mathrm{hPa}\right.$ or better) at temperatures $50^{\circ} \mathrm{C}, 80^{\circ} \mathrm{C}, 120^{\circ} \mathrm{C}$, and $160{ }^{\circ} \mathrm{C}$ for $30 \mathrm{~min}$ each and measured after each annealing step. Individual samples were annealed up to $50 \mathrm{~h}$ at $160{ }^{\circ} \mathrm{C}$ to examine whether the spin density fully recovers.

\section{RESULTS}

\section{A. Sample structure and ESR}

As a signature for the structure of the TFS material prepared by PECVD with different silane concentrations (SCs), we use the ratio of the Raman-scattering contributions from the crystalline vs amorphous phase. ${ }^{63,67}$ In Fig. 1(a) the Raman intensity ratio $I_{C}^{\mathrm{RS}}$ of the investigated intrinsic material is plotted vs the silane concentration. Note the logarithmic $x$-axis scale. $I_{C}^{\mathrm{RS}}$ decreases with increasing silane concentra- 
(a)

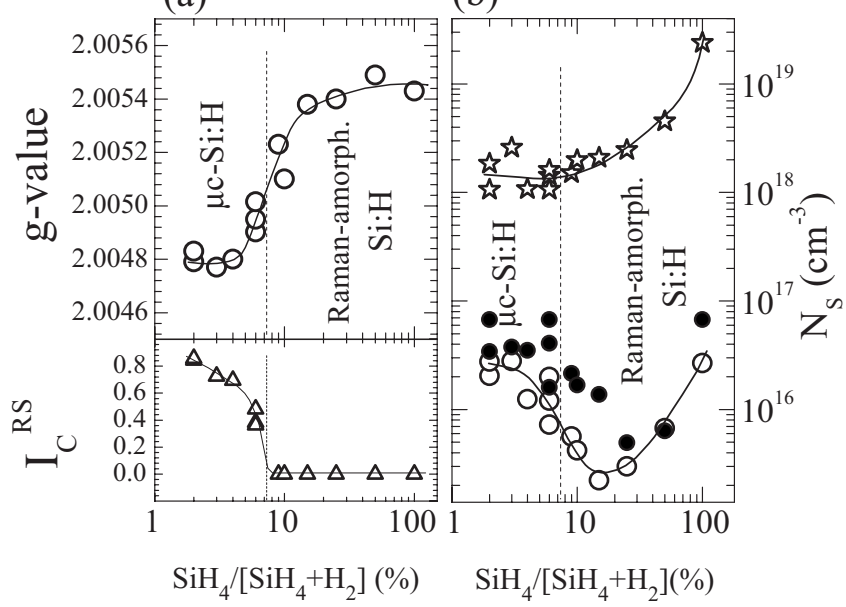

FIG. 1. Properties of the investigated undoped thin-film silicon samples as a function of the silane concentration during deposition. (a) $g$ value of the ESR resonance and Raman intensity ratio $I_{C}^{\mathrm{RS}}$. (b) Spin density $N_{S}$ after deposition (empty circles), after electron irradiation (stars), and after annealing at $160^{\circ} \mathrm{C}$ (black circles).

tion (SC). For $\mathrm{SC}>6 \%$, no crystalline Raman peak is observed. We shall call such material "Raman amorphous" to indicate that although the material shows an amorphous Raman signal only, the structure could still be different in the "amorphous" silicon prepared with different SC. The border between Raman amorphous and microcrystalline structure is indicated by the dashed line in Fig. 1. Evidence for possible structure differences comes from the small shift in the Raman line peak position and, as also plotted in Fig. 1(a), from the $g$ values of the ESR line of the material.

The $g$ value, which is in general a tensor, is a characteristic quantity for a paramagnetic defect. It is determined by the energy of the defect and the interaction with its microscopic environment. It is calculated from the resonance condition

$$
h v=g \mu_{B} B,
$$

where $h$ is the Planck constant, $v$ is the microwave frequency, $\mu_{B}$ is the Bohr magnetron, and $B$ is the magnetic field flux. The measured $g$ value is the zero crossing of the first derivative of the ESR line in the investigated material attributed to dangling bonds in intrinsic TFS. It was already found earlier ${ }^{10,28,29}$ that the $g$ values of the resonance in thinfilm silicon not only changes considerably between $a-\mathrm{Si}: \mathrm{H}$ and $\mu c-\mathrm{Si}: \mathrm{H}$ (from $g=2.0054$ to $g=2.0048$ ) but also shows a small but distinct and reproducible variation within the Raman-amorphous region $(\mathrm{SC}=8 \ldots 30 \%)$. The observed continuous variation of the $g$ value indicates a change in the defect structure or defect energy already within the Ramanamorphous material and not surprisingly when going from fully amorphous to highly crystalline material.

The corresponding spin densities before and after irradiation and after the annealing step at $160^{\circ} \mathrm{C}$ are shown in Fig. 1(b). The spin density for the as-deposited samples [open circles in Fig. 1(b)] shows a minimum for Raman-amorphous material prepared between $\mathrm{SC}=10$ and $30 \%$. Amorphous ma- (a)

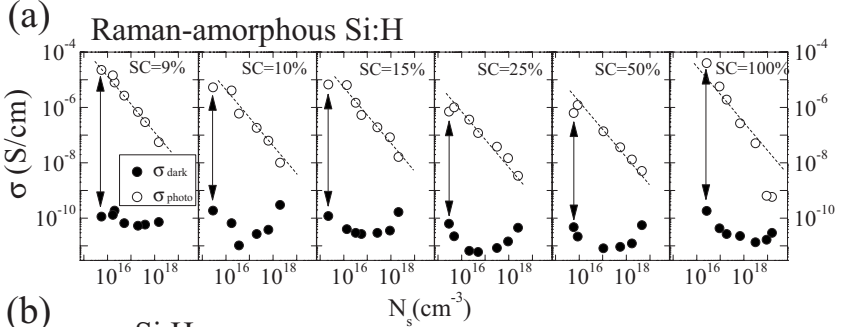

(b)

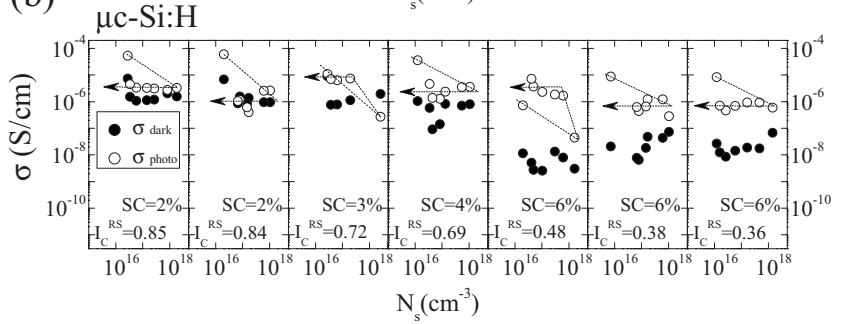

FIG. 2. Dark and photoconductivity of (a) Raman amorphous and (b) microcrystalline samples prepared with different SC as a function of the spin density $N_{S}$ obtained after deposition (left), after irradiation (right), and after individual annealing steps. The dashed line in (a) indicates a slope $m=-1$ in the relationship $\sigma_{\mathrm{ph}} \propto N_{S}^{m}$. The dashed arrows in (b) indicate the sequence of measurements: after deposition, after irradiation, and after annealing steps with increasing temperature.

terial prepared without hydrogen dilution shows higher spin densities. On the microcrystalline side, the spin density increases with decreasing SC, i.e., is highest for material with the highest crystalline volume fraction $I_{C}^{\mathrm{RS}}$. These results are in good agreement with earlier reports. ${ }^{11,68}$ After irradiation (open stars), the spin density increases by up to 3 orders of magnitude. The highest values of above $10^{19} \mathrm{~cm}^{-3}$ are found for amorphous material prepared with $\mathrm{SC}=100 \%$. For the microcrystalline material, the spin density after irradiation is in the range $1-2 \times 10^{18} \mathrm{~cm}^{-3}$. Overall the highest final spin density is found for material which had the highest initial spin density both for Raman-amorphous and for microcrystalline materials. With stepwise annealing up to $160{ }^{\circ} \mathrm{C}$ (closed circles), the spin density can be restored to its initial values. ${ }^{28,29}$ However, in general only a final annealing step considerably longer than $30 \mathrm{~min}$ results in complete defect recovering, as was confirmed for selected samples. Routinely, we have used a final annealing time of $30 \mathrm{~min}$, so a small difference between the initial and the final states remains. All data in Fig. 1 are obtained from the powder samples. In all cases, the material on glass substrates has been irradiated in parallel and similarly annealed. The following data on conductivity will be from those samples.

\section{B. Dark conductivity and photoconductivity in intrinsic thin-film silicon material}

We combine data of the conductivity measured on films with the spin density measured on correspondent powder samples to evaluate the role of defects on the charge carrier transport and recombination. Later we will show the spindensity correlation to a subgap absorption determined by CPM spectroscopy in the different TFS materials. In Fig. 2 
dark $\left(\sigma_{d}\right)$ conductivity and photo $\left(\sigma_{\mathrm{ph}}\right)$ conductivity are plotted vs spin density $N_{S}$. The results are separated into two groups: Fig. 2(a) for Raman-amorphous material and Fig. 2(b) for microcrystalline material.

Each graph in Fig. 2 is a sequence of measurements that starts at the lowest spin density (as-deposited state). The next measurement point is the one at the highest spin density (after irradiation). Then the samples are annealed stepwise resulting in the reduction in the spin density finally close to the initial values. In Fig. 2(b) this sequence is indicated by a dashed arrow. Spin density and dark and photoconductivities are measured after each annealing step.

Let us consider the Raman-amorphous samples prepared with $\mathrm{SC}=9 \ldots 100 \%$ first [Fig. 2(a)]. As a function of increasing $N_{S}$, the photoconductivity decreases almost perfectly according to $\sigma_{\mathrm{ph}} \propto N_{S}^{-1}$ over more than 2 orders of magnitude. All dashed lines in Fig. 2(a) show such an $m=-1$ slope. Only for spin densities above $10^{19} \mathrm{~cm}^{-3}$ obtained in the SC $=100 \%$ material there is a clear deviation from this behavior. This material approaches the $\sigma_{\mathrm{ph}} \propto N_{S}^{-1}$ relationship only after the second annealing step at $80{ }^{\circ} \mathrm{C}$ when $N_{S}$ is reduced to about $10^{18} \mathrm{~cm}^{-3}$. In every sample, $\sigma_{\mathrm{ph}}$ returns close to the initial value at the final annealing step.

Different from the photoconductivity, the dark conductivity in the material is little affected by the electron bombardment in all Raman-amorphous material. It remains between $10^{-10}$ and $10^{-11} \mathrm{~S} / \mathrm{cm}$ for the whole range of $N_{S}$. Nevertheless, it is interesting to note that in most cases the dark conductivity is the highest for the initial state and for the state after irradiation. Considering the indications for structural differences in the amorphous materials as indicated by the ESR $g$ values and spin densities (Fig. 1) or the shifts in the Raman peak energy, we do not observe any systematic differences in the dependence of the dark and photoconductivities on the spin density in all Raman amorphous materials.

The corresponding data for the dark conductivity and photoconductivity values versus the spin density in intrinsic $\mu c-\mathrm{Si}: \mathrm{H}$ samples prepared with $\mathrm{SC}=2-6 \%$ are shown in Fig. 2(b). The sequence from the as-deposited to the irradiated state and then through the different annealing steps is indicated by a dashed arrow in each graph. The behavior of $\sigma_{\mathrm{ph}}$ in $\mu c$-Si: $\mathrm{H}$ samples is distinctly different from the one in the Raman-amorphous material. In all samples, we observe a decay of $\sigma_{\mathrm{ph}}$ between the as-deposited and the irradiated state of approximately 1 order of magnitude only. In most cases, $\sigma_{\mathrm{ph}}$ does not recover with annealing. It remains on the level obtained after irradiation. The decrease in $\sigma_{\mathrm{ph}}$ is considerably less than what one would expect from the $\sigma_{\mathrm{ph}}$ $\propto N_{S}^{-1}$ behavior which was observed in the amorphous material. Two samples $\left(\mathrm{SC}=3 \%\right.$ and $I_{C}^{\mathrm{RS}}=0.72 ; \mathrm{SC}=6 \%$ and $I_{C}^{\mathrm{RS}}$ $=0.48$ ) show a return of $\sigma_{\mathrm{ph}}$ after the first annealing step, but again further annealing does not affect $\sigma_{\mathrm{ph}}$ while the spin density decreases. Similarly to the amorphous samples, the dark conductivity $\sigma_{d}$ in the intrinsic $\mu c-\mathrm{Si}: \mathrm{H}$ remains almost constant upon electron bombardment and annealing.

\section{Dark conductivity and photoconductivity in thin-film silicon material doped with phosphorus}

The charge carrier concentration in $\mu c-\mathrm{Si}: \mathrm{H}$ can be very effectively controlled by doping. Doping with phosphorous

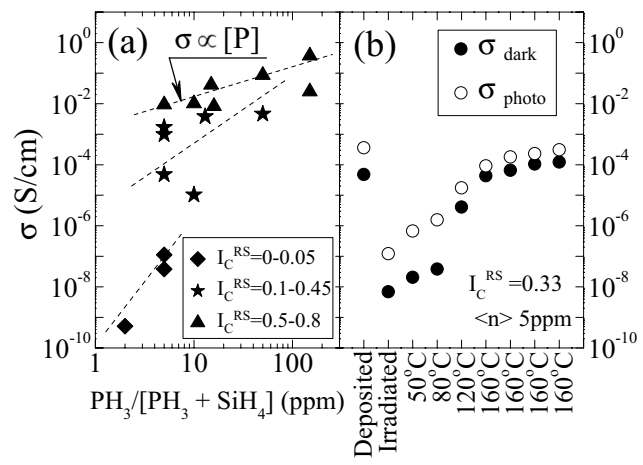

FIG. 3. (a) The dark conductivity of $n$-doped $\mu c$-Si:H samples with different ranges of $I_{C}^{\mathrm{RS}}$ (low, medium, or high) as a function of the donor doping level. The dashed lines are guides for the eyes. (b) Dark conductivity and photoconductivity of one of the $\mu c-\mathrm{Si}: \mathrm{H}$ samples with medium crystallinity versus the sample history.

(P) shifts the Fermi level toward the conduction band and the dark conductivity strongly depends on the concentration of $\mathrm{P}$ and the microstructure of the samples. ${ }^{69-72}$ The phosphorousdoped samples used here covered the whole range of crystalline volume fractions including samples with very low $I_{C}^{\mathrm{RS}}=0.05$ and two samples where the crystalline Raman peak was not resolved. Therefore, these samples are considered Raman amorphous. The maximum phosphorous concentration was chosen such that the dangling-bond density achieved during electron bombardment can shift the Fermi level significantly from its as-deposited state. The defects in the gap of $a-\mathrm{Si}: \mathrm{H}$ and $\mu c-\mathrm{Si}: \mathrm{H}$ created by electron bombardment were shown to change the photoconductivity in the intrinsic material and are expected to change the dark conductivity in the doped material as well because their occupation with electrons will change the electron concentration in the conducting states and thereby shift the Fermi energy toward midgap. In the following, the effect of the defects created by electron bombardment on the dark and photoconductivities is studied to get additional insight into the importance of defects and their charge state on the recombination also in doped material.

The influence of doping on the conductivity in the asdeposited state is illustrated in Fig. 3(a) where the dark conductivity at room temperature is shown versus the concentration of $\mathrm{PH}_{3}$ in the deposition gas mixture. The dark conductivity increases with increasing doping level, but it is obvious that the crystalline volume fraction has a significant influence on the magnitude of the conductivity and the changes in conductivity with doping level.$^{69-72}$ In the graph, the data are separated in three groups: nearly amorphous material, $\mu c-\mathrm{Si}: \mathrm{H}$ with medium crystallinity, and highly crystalline $\mu c-\mathrm{Si}: \mathrm{H}$. For the material with highest crystallinity, the highest conductivity is obtained and the conductivity is proportional to the concentration of $\mathrm{PH}_{3}$ in the gas phase. This result is very similar to those obtained in earlier studies $^{69,72}$ and by comparison of the magnitude of the conductivity values, a doping efficiency close to unity can be deduced when a mobility of $1 \mathrm{~cm}^{2} \mathrm{~V}^{-1} \mathrm{~s}^{-1}$ is assumed. With decreasing crystallinity, the conductivity decreases at a given doping level and the conductivity is no longer proportional to the concentration of $\mathrm{PH}_{3}$ in the plasma. 


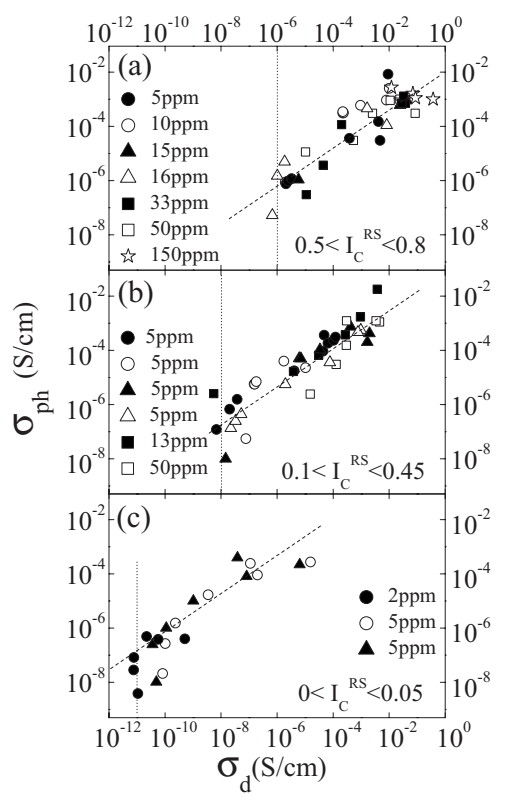

FIG. 4. Photoconductivity vs dark conductivity of the $n$-doped $\mu c-\mathrm{Si}: \mathrm{H}$ and $a-\mathrm{Si}: \mathrm{H}$ samples after different annealing steps for material with (a) high, (b) medium, and (c) low or no crystallinity. Each graph contains data from several samples of different phosphorous doping concentrations. The dashed lines correspond to a relationship $\sigma_{\mathrm{ph}} \propto \sigma_{d}^{0.7}$. The vertical dotted lines indicate the level of the dark conductivity in intrinsic samples of the given crystallinity range after irradiation.

The dark conductivity along with the photoconductivity for the complete experimental sequence for one of the $n$-type $\mu c$-Si: $\mathrm{H}$ samples with a medium crystalline volume fraction of 0.33 is presented in Fig. 3(b) as an example. After the electron bombardment (irradiated), a decrease of both $\sigma_{d}$ and $\sigma_{\mathrm{ph}}$ by several orders of magnitude is observed with respect to the as-deposited state. Subsequent annealing at the indicated temperatures leads to the stepwise recovery of both values, but a recovery of $\sigma_{d}$ and $\sigma_{\mathrm{ph}}$ close to their initial values is achieved only for long annealing times at $160{ }^{\circ} \mathrm{C}$. The dependence of the recovery is different for $\sigma_{d}$ and $\sigma_{\mathrm{ph}}$, i.e., at annealing temperatures up to $80^{\circ} \mathrm{C}$ the ratio of the dark and photoconductivities is much larger than at higher annealing temperatures.

The photoconductivity of phosphorous doped $a$-Si:H has a characteristic dependence on the dark conductivity ${ }^{73-77}$ which has been interpreted as an effect of the change in the occupation of dangling bonds that act as recombination centers. To investigate whether this holds also in $\mu c-\mathrm{Si}: \mathrm{H}, \sigma_{\mathrm{ph}}$ is plotted vs $\sigma_{d}$ in Fig. 4 for all samples at the different stages of annealing. The data are separated into three graphs according to the crystallinity of the material as in Fig. 3(a). Note that all graphs have the same scale.

In samples with doping level of not more than $16 \mathrm{ppm}$ $\mathrm{PH}_{3}$, the electron bombardment results in reduction of $\sigma_{d}$ to the level of intrinsic material (shown with a dashed line in each graph in Fig. 4). The decrease in the dark conductivity is accompanied by a remarkable reduction in the photoconductivity. The relation may be roughly approximated as $\sigma_{\mathrm{ph}}$ $\propto \sigma_{d}^{0.7}$. Such a correlation of $\sigma_{d}$ and $\sigma_{\mathrm{ph}}$ is observed for up to
7 orders of magnitude of $\sigma_{d}$ no matter whether $\sigma_{d}$ is determined by different doping concentration or different stages of annealing. Interestingly, the relation seems to be not affected by the microstructure. However, at high $\sigma_{d}$ the dependence of $\sigma_{\mathrm{ph}}$ on $\sigma_{d}$ becomes weaker. On the other hand, for $\sigma_{d}$ closer to the intrinsic value, this dependence becomes stronger and in some cases $\sigma_{\mathrm{ph}}$ still changes even when the dark conductivity is almost constant. Although the general trend for the dependency of $\sigma_{\mathrm{ph}}$ on $\sigma_{d}$ is very similar for the Raman-amorphous material (Fig. 4 bottom) and the material with high crystallinity (Fig. 4 top), the ratio of $\sigma_{\mathrm{ph}}$ and $\sigma_{d}$ is about 3 orders of magnitude larger in the former than in the latter. An intermediate behavior is found in $\mu c-\mathrm{Si}: \mathrm{H}$ with medium crystalline volume fraction (Fig. 4 middle).

To summarize the effect of electron bombardment on $\mu c-\mathrm{Si}: \mathrm{H}$ in comparison to $a-\mathrm{Si}: \mathrm{H}:$ the influence of the radiation induced defects on the photoconductivity and dark conductivity of undoped material is different for $a-\mathrm{Si}: \mathrm{H}$ and $\mu c-\mathrm{Si}: \mathrm{H}$. For $a-\mathrm{Si}: \mathrm{H} \sigma_{\mathrm{ph}}$ is inversely proportional to $N_{S}$ whereas it is not in $\mu c-\mathrm{Si}: \mathrm{H}$. On the other hand, in both materials, the photoconductivity depends on the dark conductivity in a similar way, i.e., the charge state of the defects shows the largest effect on $\sigma_{\mathrm{ph}}$.

\section{Constant photocurrent measurements}

Photocurrent spectroscopy in the CPM has been measured on the irradiated material for two reasons. (i) The prospect to determine the defect density directly on the substrate samples where $\sigma_{d}$ and $\sigma_{\mathrm{ph}}$ are measured in view of the difficulties to evaluate ESR spectra on the irradiated samples because of the heavily dominating $E^{\prime}$ center resonance of defects generated in the substrate. (ii) To evaluate the applicability of CPM for the determination of the defect absorption contribution in $\mu c-\mathrm{Si}: \mathrm{H}$ with its heterogeneous structure and its modified optical-absorption characteristic with respect to $a-\mathrm{Si}: \mathrm{H}$.

CPM spectra were measured after each annealing step together with the conductivity measurements. The results for $a-\mathrm{Si}: \mathrm{H}$ and $\mu c-\mathrm{Si}: \mathrm{H}$ samples are presented in Fig. 5. All spectra are normalized at the onset of saturation of the CPM signal which is at a photon energy of around $1.75 \mathrm{eV}$ for $a-\mathrm{Si}: \mathrm{H}$ and $1.35 \mathrm{eV}$ for $\mu c-\mathrm{Si}: \mathrm{H}$. The normalization procedure is based on the assumption that the band-to-band absorption is not affected by the defect density in particular sample. A variation in the band-to-band absorption would be related to the change in the material structure but no evidence for such variation is found in the study. We compare relative variation in CPM spectra of each particular sample. At $1.75 \mathrm{eV}$, the CPM signal is a superposition of the subgap and band-to-band signals. In case of highest defect densities, the subgap component has to be subtracted from the signal at $1.75 \mathrm{eV}$ for correct normalization. For the sake of clarity in Fig. 5, we present the spectra normalized as is without this correction. However such correction with extrapolated values for the subgap signal was performed for the values of CPM subgap absorption for comparison with $N_{S}$.

The results are separated in two groups: for Ramanamorphous material [Fig. 5(a)] and for $\mu c-\mathrm{Si}: \mathrm{H}$ [Fig. 5(b)]. 
(a) Raman- amorphous $\mathrm{Si}: \mathrm{H} \quad-$ - As-deposited

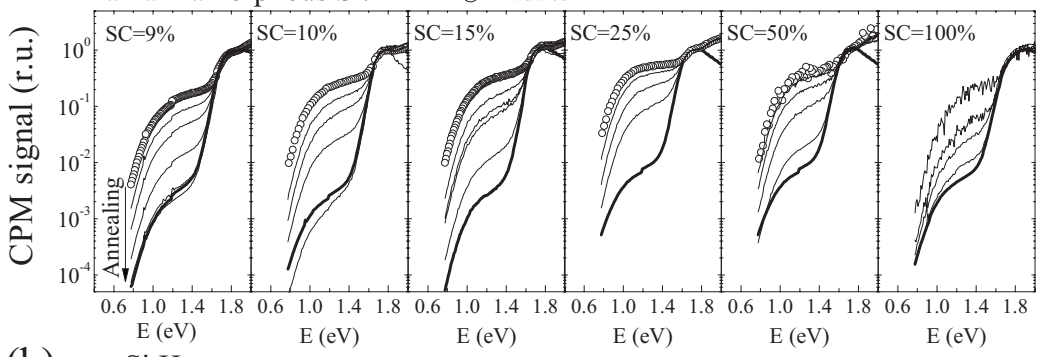

(b) $\mu \mathrm{c}-\mathrm{Si}: \mathrm{H}$

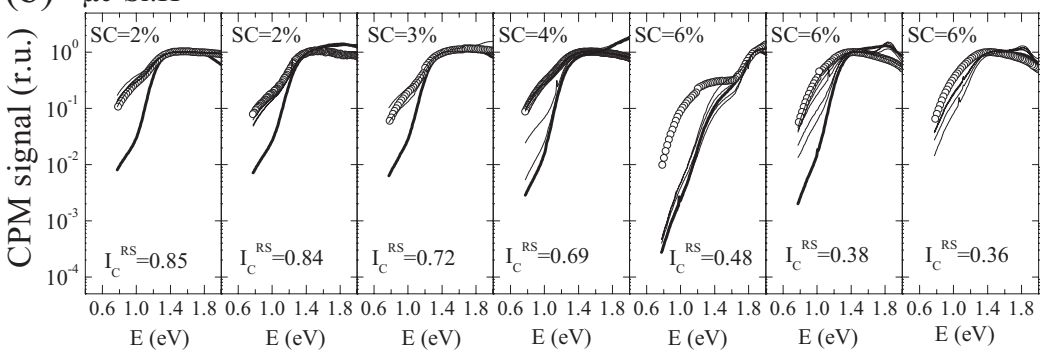

FIG. 5. CPM spectra of (a) Ramanamorphous samples and (b) the $\mu c-\mathrm{Si}: \mathrm{H}$ samples measured after deposition (bold lines), after electron bombardment (empty circles) and between annealing steps (thin lines).
Spectra are shown for the samples in the as-deposited state, after electron bombardment and after various annealing steps. The sample with $\mathrm{SC}=100 \%$ could not be measured with CPM directly after irradiation because of extremely low photoconductivity. Only after annealing at $80{ }^{\circ} \mathrm{C}$ the $\mathrm{CPM}$ response was recovered.

The Raman-amorphous material is considered first [Fig. 5(a)]. For all samples we see a very good qualitative agreement with the expectations: The height of the absorption shoulder below the absorption edge (e.g., at $1.2 \mathrm{eV}$ ) increases by about 2 orders of magnitude between the asdeposited state and the irradiated state. With annealing, the CPM spectrum recovers to its initial shape, indicating a complete annealing of the generated defects. There are no indications for changes in the slope of the absorption edge.

Such a clear qualitative development of the CPM spectra upon irradiation and annealing is not found in the $\mu c-\mathrm{Si}: \mathrm{H}$ samples [Fig. 5(b)]. We assume that the defect absorption contribution in $\mu c-\mathrm{Si}: \mathrm{H}$ is located at photon energies of 0.9 $\mathrm{eV}$ and below. After irradiation, we observe a certain increase in the subgap absorption shoulder of about 1 order of magnitude for all $\mu c$-Si:H samples. But a systematic and monotonic recovery of the spectra upon annealing was not observed. The spectra of the highly crystalline material $(\mathrm{SC}=2 \ldots 3 \%)$ do not recover at all upon annealing. The spectra of less crystalline $\mu c-\mathrm{Si}: \mathrm{H}(4 \ldots 6 \%)$ do recover close to the as-deposited state but not in the gradual manner as observed in $a-\mathrm{Si}: \mathrm{H}$. In addition, material with presumably similar structural properties $(\mathrm{SC}=6 \%)$ shows quite different behavior.

In order to quantify the changes in the subgap absorption upon irradiation and annealing, we have determined the absorption values (in relative units) at $1.2 \mathrm{eV}$ for $a-\mathrm{Si}: \mathrm{H}$ and $0.9 \mathrm{eV}$ for $\mu c-\mathrm{Si}: \mathrm{H}$. These values are plotted vs the spin densities normalized to the spin density of the as-deposited material in Figs. 6(a) and 6(b). This presentation allows one to evaluate the proposed proportionality between the defect absorption determined from CPM with the spin density measured with ESR over a wide range of spin densities, irrespec- tive of the initial spin densities in the individual samples. Note that both figures are plotted with the same axes and have the same scale.

For the Raman-amorphous materials [Fig. 6(a)], we find a good 1:1 relationship between CPM determined defect absorption and spin density for individual samples and for all samples in general for the defect density range $N_{S} / N_{S}$ init $\leq 100$. In absolute spin densities this corresponds to about $10^{18} \mathrm{~cm}^{-3}$ for the upper limit. For higher values of spin densities of $N_{S} / N_{S}$ init $\geq 100$, there is a deviation from this linear relationship. We further note that there is no discernible difference between the various Raman-amorphous materials concerning their behavior in the CPM vs ESR data comparison.

For the $\mu c-\mathrm{Si}: \mathrm{H}$ material, an analytic relationship between defect absorption determined from CPM and spin density is not visible [Fig. 6(b)]. This was already obvious from the spectra in Fig. 5. Individual samples do show a correlation between the two quantities but the dynamic range is much reduced because of the small change in the defect absorption shoulder and the slope of the relationship is differ-

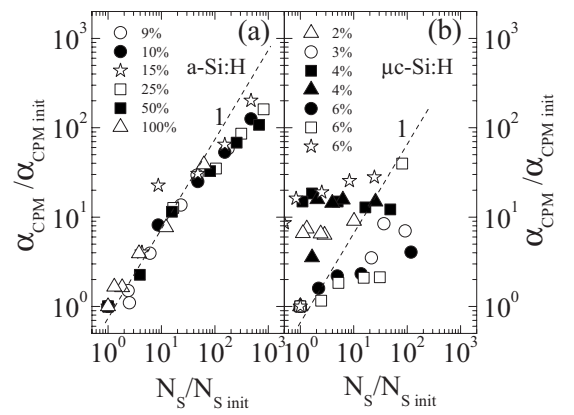

FIG. 6. The relative change in the defect absorption shoulder in the CPM spectra $\left(\alpha_{\mathrm{CPM}} / \alpha_{\mathrm{CPM} \text { init }}\right)$ as a function of the relative change in spin density $\left(N_{S} / N_{S}\right.$ init $)$ for (a) the Raman amorphous samples where $\alpha_{\mathrm{CPM}}$ is taken at $E=1.2 \mathrm{eV}$ and (b) for the microcrystalline samples where $\alpha_{\mathrm{CPM}}$ is taken at $E=0.9 \mathrm{eV}$. The dashed line indicates direct proportionality $\alpha_{\mathrm{CPM}} \propto N_{S}$. The values in legend are SC. 
ent for all samples. A general trend cannot be extracted and determination of a single relationship of $\alpha \propto N_{S}$ is ambiguous.

\section{DISCUSSION}

In the present work, we have investigated the influence of defects in thin-film silicon material all the way from highly crystalline to amorphous structure on (i) dark and photoconductivities in intrinsic material, (ii) the Fermi-level shift and resulting change in the dark and photoconductivities in $n$-doped material, and (iii) photoconductivity spectroscopy in the CPM mode with the aim to evaluate the defect density.

In the following we shall discuss these three topics. To start with, we want to comment briefly on the assumption that the defect generation and annealing process (i) mainly changes the dangling-bond density and (ii) these dbs are the main deep defects in the mobility gap which interact with charge carriers.

From numerous experiments on $a-\mathrm{Si}: \mathrm{H}$ and $\mu c-\mathrm{Si}: \mathrm{H}$ with electron irradiation and annealing, ${ }^{28-31}$ we can conclude that the irradiation and annealing sequence is a reversible process such that after several bombardment and annealing procedures the material has returned to the as-deposited state. A further general observation is that the increase in the electron-spin-resonance signal is always at the $g$ values which are also present in the as-deposited state. ${ }^{15,28-31} \mathrm{We}$ therefore conclude that the electron beam generates additional "intrinsic" defects.

For the $a-\mathrm{Si}: \mathrm{H}$ material, both intrinsic and doped identification of the electron-beam generated defects as dangling bonds is fairly obvious from the $g$ values and line parameters observed. The additional dbs are considered to interact with carriers in the same way as the db defects in the as-deposited state. All experiments performed so far are in very good agreement with this identification. For Raman amorphous material prepared with high hydrogen dilution, which shows a small shift of the $g$ value but generally very similar relationships between the defects and transport properties as the "fully" amorphous material, it is safe to conclude similarly.

For $\mu c-\mathrm{Si}: \mathrm{H}$, the situation is more complicated. Asdeposited intrinsic material shows an ESR signal which can be interpreted as a superposition of two resonances or as a powder pattern from an anisotropic defect. Identification with dangling bonds is plausible but the location of the defects in crystalline or amorphous regions or at grain boundaries is not known. From previous studies on electronbombarded material, ${ }^{15}$ we conclude that the signal is a superposition of two resonances at $g=2.0043$ and $g$ $=2.0052$ and the electron irradiation generates $\mathrm{db}$ defects preferably at a $g$ value of $g=2.0052$. We further conclude from studies on doped $\mu c-\mathrm{Si}: \mathrm{H}$ material that the spin defects represent the majority of deep defects interacting with charge carriers in $\mu c-\mathrm{Si}: \mathrm{H}^{10}$ So there is a good evidence that the two assumptions are valid also in the $\mu c-\mathrm{Si}: \mathrm{H}$ material and therefore in all thin-film silicon samples investigated here.

\section{CONDUCTIVITY}

In the following, we use the common description of the conductivity in amorphous silicon as given, e.g., in Refs. 1 and 78, in order to discuss the results obtained in our thinfilm silicon samples in the whole range of composition because previous work gave strong evidence that the electronic transport in microcrystalline silicon exhibits many similarities with that in amorphous silicon. ${ }^{69,70}$ The dark conductivity $\sigma_{d}$ can then be written as

$$
\sigma_{d}=\sigma_{0} \exp \left[\left(E_{C}-E_{F}\right) / k T\right]
$$

where $E_{F}$ is the Fermi energy, $E_{C}$ is the mobility edge for electrons (conduction band edge), $k$ is the Boltzmann constant, and $T$ is the temperature in Kelvin. The conductivity prefactor $\sigma_{0}$ is the product of the carrier mobility, the effective density of states, and the electron charge. Its value is close to $150 \mathrm{~S} / \mathrm{cm} .{ }^{1,75}$ Accordingly, the energy difference between the Fermi energy and the mobility edge at a given temperature can be calculated from the dark conductivity at a given temperature

$$
E_{C}-E_{F}=k T \ln \left(\sigma_{d} / \sigma_{0}\right) .
$$

\section{A. Conductivity in intrinsic $a-\mathrm{Si}: \mathbf{H}$}

A first important finding is that the dark conductivity in the intrinsic Raman-amorphous material is little affected by the electron bombardment. This means that the Fermi level does not change from its position $\left(E_{F}-E_{C} \approx 0.72 \mathrm{eV}\right)$ which implies that the position is already at midgap. Based on Eq. (3) we can estimate a maximum shift of $E_{F}$ of $0.08 \mathrm{eV}$ over the whole range of defect concentration. Consequently the charge state of defects in the gap which could influence the recombination lifetime of excess carriers should not change. The recombination rate should therefore be mainly affected by the density of defects in the gap which can be expressed by the inverse carrier lifetime proportional to the density of neutral defects $N_{S}{ }^{1}$

$$
\frac{1}{\tau} \propto N_{S}
$$

Our experimental results compare very well with this dependency over more than 3 orders of magnitude of $N_{S}$ if we consider the range covered by all our samples. This is shown in Fig. 7(a) where all $\sigma_{\mathrm{ph}}$ vs $N_{S}$ curves from Fig. 2(a) are normalized to the level given by the $\mathrm{SC}=9 \%$ sample. This allows to compare the dependency directly irrespective of the absolute photoconductivity level of the individual sample.

With this result, the proposed relationship is now confirmed experimentally on a number of different samples over a much wider range than documented so far. To illustrate this, we compare our data (sample $\mathrm{SC}=9 \%$ ) with the data of earlier works $2,33,35,44,46$ in Fig. 7(b). Those studies individually cover a smaller range of defect densities and some show considerable deviation from the $\sigma_{\mathrm{ph}} \propto N_{S}^{-1}$ dependency. ${ }^{33,35,46}$ We cannot discuss possible reasons for the deviations from the proposed relationship (1) in the earlier reports. However, it is tempting to assume the leveling off at high ${ }^{33,44,46}$ and low $^{35} N_{S}$ values to be a result of experimental problems with uncontrolled defect annealing or incomplete and inhomogeneous defect generation. Consequently, the experimental pro- 


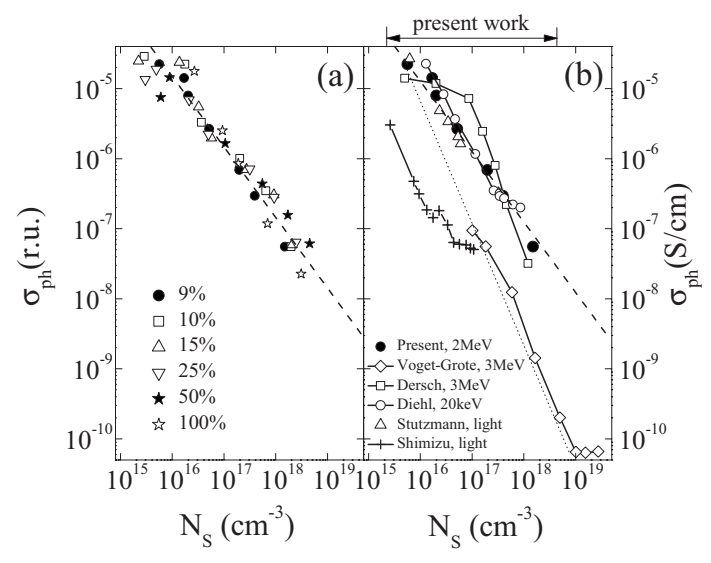

FIG. 7. (a) Summary of the photoconductivity data presented in Fig. 2(a). The values in legend are SC. For details see text. (b) A comparison of the dependence of $\sigma_{\mathrm{ph}}$ on $N_{S}$ obtained in our experiment with earlier reported results achieved with different methods: $\mathrm{MeV}$ electron bombardment [Voget-Grote (Ref. 33) and Dersch (Ref. 35)], keV electron bombardment [Diehl (Ref. 44)], and light soaking [Stutzmann (Ref. 2) and Shimizu (Ref. 46)]. The dashed line and dotted line indicate the relationship $\sigma_{\mathrm{ph}} \propto N_{S}^{-1}$ and $\sigma_{\mathrm{ph}}$ $\propto N_{S}^{-2}$, respectively. The whole region of $N_{S}$ covered by different samples in our experiment [Fig. 7(a)] is indicated on top of the figure.

cedure described here, with the maintenance of the cold chain between irradiation and measurement is considered the key for the successful demonstration of relationship (1) in $a-\mathrm{Si}: \mathrm{H}$.

In summary our data experimentally confirm with good statistics from several samples the model for direct capture as the dominating process for recombination of excess carriers in intrinsic amorphous hydrogenated silicon over more than 3 orders of magnitude. The data give a sound experimental basis for the treatment of recombination via neutral dangling bonds in device simulation of solar cells with amorphous silicon absorber layers.

The findings prove the fundamental importance of the defect density for the material quality in intrinsic amorphous silicon in terms of charge carrier lifetime. In particular, the data support earlier suggestion that a further improvement should result from further reduction in the defect density.

\section{B. Conductivity in intrinsic $\mu c-\mathrm{Si}: \mathbf{H}$}

Though $N_{S}$ in $\mu c-\mathrm{Si}: \mathrm{H}$ decreases with annealing stepwise very similarly to $a$-Si:H (Refs. 15,28 , and 31 ), the relationship between defects and $\sigma_{\mathrm{ph}}$ in the intrinsic $\mu c$-Si: H material clearly deviates from the behavior described above for the amorphous material. We point out three important findings. (i) The initial decay of $\sigma_{\mathrm{ph}}$ is considerably less than one would expect from a $\sigma_{\mathrm{ph}} \propto N_{S}^{-1}$ behavior. (ii) The $\sigma_{\mathrm{ph}}$ is largely independent from $N_{S}$ when spin defects are annealed. This is true at least for the observed range of $N_{S}=1$ $\times 10^{16}-3 \times 10^{18} \mathrm{~cm}^{-3}$. (iii) The material shows no consistent behavior, in some samples the $\sigma_{\mathrm{ph}}$ recovers, in some it does not.

At least the behavior of dark conductivity $\sigma_{d}$ as a function of irradiation and annealing fits in the expected picture of generation of defects near midgap and of intrinsic transport behavior of the material in the as-deposited state, after irradiation and after all annealing steps. The $\sigma_{d}$ changes only little in nonsystematic way and the resulting shift of the Fermi level is not more than $0.09 \mathrm{eV}$ estimated from Eq. (3b). Also worth mentioning is that from the invariance of $\sigma_{d}$, one would conclude that there are no changes in the transport paths of charge carriers in thermal equilibrium.

In contrast to the dark conductivity, the behavior of $\sigma_{\mathrm{ph}}$ vs $N_{S}$ is puzzling. To explain these findings, one can excuse almost all by the heterogeneous structure composition of the material and by the difficulty to reproduce this structure near the phase transition (here $\mathrm{SC}=6 \%$ ). This seems unsatisfactory. We want therefore to discuss at least a possible explanation which, however, has to remain qualitative as any quantitative model seems too ambitious in view of the complexity of the problem.

The decrease in $\sigma_{\mathrm{ph}}$ is considerably less than what one would expect from the $\sigma_{\mathrm{ph}} \propto N_{S}^{-1}$ behavior which was observed in the amorphous material. Apparently the electron irradiation-induced spin defects do not interact with the excess charge carriers in a conventional way in $\mu c-\mathrm{Si}: \mathrm{H}$. An intuitive suggestion would be that the defects are spatially or/and electrically separated from the transport channels or are clustered in regions which can be by passed by charge carriers. Therefore even at high net defect density, there is a possibility for the excess carriers to drift through the regions where the defect density is locally low. As a matter of speculation, this situation may be described as follows. Defects are generated homogeneously in the material in the crystalline and amorphous phase. But the stability of the radiationinduced dangling bonds in the crystalline and amorphous regions could differ significantly. In general, the temperature of irradiation (around $100 \mathrm{~K}$ ) is already sufficient for the active diffusion and annealing of the primary defects in crystalline silicon. ${ }^{79}$ Therefore even at equal defect generation rate, the amorphous regions will contain more defects. This process will continue at further annealing steps. As a result, we have a high local density of defects at the grain and column boundaries which, however, can be small in absolute numbers. The grain-boundary defects are stable up to annealing temperatures of $160{ }^{\circ} \mathrm{C}$ and therefore result in an irreparable damage of the grain boundaries.

In summary, the influence of deep defects on the photoconductivity in $\mu c-\mathrm{Si}: \mathrm{H}$ is weaker than in amorphous silicon but still evident. Defects at grain or column boundaries in $\mu c-\mathrm{Si}: \mathrm{H}$ will determine the material quality in agreement with earlier interpretations on the relationships between deposition process, structure and defects, and electronic quality and device performance. ${ }^{71,80,81} \mathrm{An}$ analytic relationship such as in the case of $a-\mathrm{Si}: \mathrm{H}$ is not found. The heterogeneous structure composition in $\mu c-\mathrm{Si}: \mathrm{H}$ and different defect annealing kinetics in the crystalline and amorphous regions, respectively, is suggested to play an important role here providing inhomogeneous distribution of the defects in $\mu c$-Si: $\mathrm{H}$ after irradiation.

\section{Conductivity in $\mu c-\mathrm{Si}: \mathrm{H}$ doped with $\mathrm{P}$}

In $n$-doped $\mu c-\mathrm{Si}: \mathrm{H}, \sigma_{d}$ and $\sigma_{\mathrm{ph}}$ are similarly sensitive to the irradiation and annealing as in $a-\mathrm{Si}: \mathrm{H}$ in contrast to the 
dependence of photoconductivity on the defect density in intrinsic $\mu c$-Si:H. In the following, we will discuss the dependence of $\sigma_{d}$ and $\sigma_{\mathrm{ph}}$ on the defect density for both types of material in detail. However, the defect density in doped samples cannot be measured directly by ESR because the occupation of $\mathrm{db}$ states (portion of neutral, paramagnetic db's) will vary considerably during experiment. On the other hand, changes in the photoconductivity due to Fermi-level shift have been reported for $a-\mathrm{Si}: \mathrm{H}$ (Refs. 73-77) and $\mu c-\mathrm{Si}: \mathrm{H} \cdot{ }^{4,82-85}$ Therefore, the dependence of $\sigma_{\mathrm{ph}}$ on $\sigma_{d}$ is used to evaluate the effect of the defect density on the recombination in doped samples.

In order to discuss the results on a semiquantitative basis, the relation between doping, carrier concentration, and dark conductivity is briefly introduced in the following. Phosphorous can be built into $\mu c-\mathrm{Si}: \mathrm{H}$ from the gas phase with an efficiency of about $50 \%$ and the doping efficiency under similar conditions as in this study is close to unity. ${ }^{10,70,86}$ In contrast in $a-\mathrm{Si}: \mathrm{H}$, the doping efficiency is a function of the doping level due to an "autocompensation effect," i.e., the creation of dangling-bond defects caused by the shift of the Fermi level $E_{F} \cdot{ }^{1}$ The results on the intrinsic $a-\mathrm{Si}: \mathrm{H}$ and $\mu c$-Si:H samples indicate that in both materials the density of defects can be changed by electron bombardment over many orders of magnitude without changing the microstructure of the material. It is assumed that this holds also for the doped samples.

The dark conductivity for a semiconductor with electrons as a majority carrier is given by $\sigma_{d}=[n] \mu e$, with $[n]$ and $\mu$ the concentration and mobility of the carriers, respectively, and $e$ the charge of electrons. As shown in Fig. 3 the doping of $\mu c-\mathrm{Si}: \mathrm{H}$ with phosphorous leads to an increase in the dark conductivity. This increase is basically due to an increase in the density of electrons in conducting states. For the highly crystalline material, the observed proportionality between the doping level and the dark conductivity shows that the concentration of electrons is basically given by the concentration of ionized phosphorous donors. This means that in this case, according to a simple expression for the carrier concentration,

$$
[n]=[P]-\left[N_{d}\right]+\left[N_{b}\right]=[P]-\left[N_{s}\right]
$$

the donor concentration $[P]$ is significantly higher than the defects which will be occupied at first. These defects are the difference between the total defect concentration $\left[N_{d}\right]$ and the net background doping $\left[N_{b}\right]$. We can safely assume that the predominant defects in our films are the dangling bonds; therefore $\left[N_{d}\right]-\left[N_{b}\right]$ equals the density of singly occupied dangling bonds $\left[N_{s}\right]$. The lowest phosphorous concentration for the highly crystalline material in this study is $5 \mathrm{ppm}$ which translates to $1.25 \times 10^{17} \mathrm{~cm}^{-3}$ for a built-in coefficient of the phosphorous of 0.5 . As there is no deviation from the proportional increase of $\sigma_{d}$ at this doping level, the upper limit for the density of electrons needed to occupy the defects is a few times $10^{16} \mathrm{~cm}^{-3}$. This value is consistent with the measured spin density of the intrinsic $\mu c-\mathrm{Si}: \mathrm{H}$ samples deposited under similar conditions which was always lower than $3 \times 10^{16} \mathrm{~cm}^{-3}$ in the as-deposited state [compare Fig. 1(b)]. The typical $p$-type doping level to compensate the $n$-type character in such material is about 1 ppm, e.g., $\left[N_{b}\right]$ $\approx 5 \times 10^{16} \mathrm{~cm}^{-3} .{ }^{71}$ Therefore, the total defect density $\left[N_{d}\right]$ in such films can be approximated to be less than $8 \times 10^{16} \mathrm{~cm}^{-3}$ : a value more than 1 order of magnitude lower than the spin density created by the electron irradiation.

For the samples with medium crystallinity $\left(0.1<I_{C}^{\mathrm{RS}}\right.$ $<0.45)$, the proportionality is not observed and for a given doping level the absolute value of the dark conductivity is lower than in the highly crystalline material. As shown in Fig. 1 and in previous work, ${ }^{30,31}$ the dangling-bond density in the intrinsic samples with medium crystallinity is not higher than in the highly crystalline material. It is therefore suggested that for medium crystallinity region where crystalline volume dominates, the reduced charge carrier mobility is the cause of the lower dark conductivity in samples with decreasing crystallinity. Indeed, Hall measurements have shown that the charge carrier mobility in $\mu c-\mathrm{Si}: \mathrm{H}$ depends on the charge carrier density and on the microstructure of the material. This effect is most pronounced at low carrier densities, ${ }^{70,87}$ e.g., for a carrier density of $2 \times 10^{16} \mathrm{~cm}^{-3}$, the carrier mobility decreases by about a factor of 5 from $I_{C}^{\mathrm{RS}}$ $\approx 70 \%$ to $I_{C}^{\mathrm{RS}} \approx 35 \% .{ }^{87}$ At even lower $I_{C}^{\mathrm{RS}}$ values, the mobility is further reduced, and the mobility is lowest in amorphous material. The strong decrease in the dark conductivity in the highly amorphous material, however, stems from the lower doping efficiency, the fairly high defect density, and the high density of band tail states which limits the concentration of mobile carriers.

\section{Dark conductivity and defects in $\mu c-\mathrm{Si}: \mathrm{H}$ doped with $\mathrm{P}$}

For a given sample the decrease of $\sigma_{d}$ can be attributed to decrease in the carrier concentration caused by an increase in the defect concentration after irradiation as shown in Fig. 3(b) for the radiation and annealing sequence. This can be semiquantitatively discussed using Eq. (5). According to Fig. 1(b) the defect density introduced by electron bombardment is about $1-2 \times 10^{18} \mathrm{~cm}^{-3}$ except for the samples with the highest $\mathrm{SC}$ which are not used for the doping series. This value for the defect concentration does not vary too much with $I_{C}^{\mathrm{RS}}$. This means that for all samples with doping concentration less than about $20 \mathrm{ppm}$, the defect density is larger than the donor concentration and the sample should exhibit a dark conductivity similar to an intrinsic sample. A comparison between Figs. 2 and 4 shows that this is indeed the case. The dark conductivity after irradiation indicated by the dashed lines in Fig. 4 compares well with the dark conductivity in the intrinsic sample irrespective whether the sample is irradiated or not. For higher doping concentration, the introduced defect density cannot fully compensate the donor concentration and therefore the carrier concentration and thus the dark conductivity remains at a higher level. Reducing the defect concentration by annealing will lead to an increase in the carrier concentration once the defect concentration is lower than the donor concentration. Using Eq. (3b) the variation of $\sigma_{d}$ can be translated into a shift of the Fermi level of about $0.39 \mathrm{eV}$ for the Raman amorphous material, for the highly crystalline material this shift is up to $0.30 \mathrm{eV}$. 
On the basis of the $\sigma_{d}$ values shown in Fig. 4, Eq. (3b) can also be used to estimate the distance of the Fermi energy from the transport energy of the charge carriers for the whole range of structure composition from fully microcrystalline to fully amorphous material. For the material with the lowest dark conductivity, i.e., the highest defects density, one obtains $E_{C}-E_{F} \approx 0.41 \mathrm{eV}$ for $I_{C}^{\mathrm{RS}}>0.5, E_{C}-E_{F} \approx 0.53 \mathrm{eV}$ for $0.1<I_{C}^{\mathrm{RS}}<0.4$, and $E_{C}-E_{F} \approx 0.75 \mathrm{eV}$ for $I_{C}^{\mathrm{RS}}<0.05$ assuming the conductivity prefactor $\sigma_{0}=150 \mathrm{~S} / \mathrm{cm}$ as determined for $a-\mathrm{Si}: \mathrm{H}$. If the dangling bonds are created close to the midgap, the Fermi energy will be located there and one can estimate an effective mobility gap $E_{\mu}$, for these material with different structural composition. In this case $E_{\mu}$ is simply twice $E_{C}-E_{F}$ and amounts to $E_{\mu} \approx 0.82 \mathrm{eV}$ for $I_{C}^{\mathrm{RS}}>0.5$, $E_{\mu} \approx 1.05 \mathrm{eV}$ for $0.1<I_{C}^{\mathrm{RS}}<0.4$, and $E_{\mu} \approx 1.51 \mathrm{eV}$ for $I_{C}^{\mathrm{RS}}$ $<0.05$. On the first sight, the value for highly crystalline material seems not unreasonable for a mobility gap because the partial disorder in this material introduces band tail states below the gap of crystalline silicon as evidenced by various experiments such as photoluminescence spectroscopy ${ }^{88}$ and time of flight. ${ }^{89}$ However, this value for the mobility gap $E_{\mu}$ is difficult to reconcile with the fairly low density of states at this energy which is dominated by defects as indicated by the CPM spectra in Fig. 5 and by subgap absorption measurements with photothermal deflection spectroscopy (PDS). ${ }^{62}$ The same difficulty is observed for the amorphous sample where $\alpha$ is too low at $E_{\mu} \approx 1.5 \mathrm{eV}$. It is therefore suggested that the mobility gap is underestimated when using a value of $\sigma_{0}=150 \mathrm{~S} / \mathrm{cm}$ which is based on the minimum metallic conductivity approach or that the defects are located above the midgap. It should be noted that similar values for $E_{\mu}$ are obtained for the intrinsic and doped materials with the same microstructure as $\sigma_{d}$ is almost equal in both cases at high defect density. The increase in the mobility gap in $\mu c-\mathrm{Si}: \mathrm{H}$ with medium crystallinity can be explained by additional barriers for charge carrier transport due to an increasing amorphous phase which add barriers for transport without changing the transport in the crystalline phase. The effective mobility gap would then be a superposition of the mobility gap and the barrier height.

\section{E. Photoconductivity and defects in $\mu c-\mathrm{Si}: \mathrm{H}$ doped with $\mathbf{P}$}

The strong correlation between the dark conductivity and photoconductivity points to the crucial role of the position of the $E_{F}$ on the photoconductivity. Such an effect is known from $a-\mathrm{Si}: \mathrm{H}$ and interpreted on the basis of the change in the occupation of recombination centers by charge carriers provided by donors (in case of $n$-type material). The electron bombardment and subsequent annealing allow to investigate (i) such a dependency for the broad range of the photoconductivity in one and the same sample and (ii) the role of the defects in a sample with the same position of the $E_{F}$ but different defect concentration. We can compare our results with those achieved previously for a series of $\mu c-\mathrm{Si}: \mathrm{H},{ }^{82}$ doped samples of $a-\mathrm{Si}: \mathrm{H}$ (Ref. 73) and $\mu c-\mathrm{Si}: \mathrm{H},{ }^{4}$ or with the degradation of the doped $\mu c-\mathrm{Si}: \mathrm{H}^{83-85}$

In Fig. 4 the dependence of $\sigma_{\mathrm{ph}}$ on $\sigma_{d}$ is shown and despite the scatter of the data a power-law behavior $\left(\sigma_{\mathrm{ph}}\right.$ $\left.\propto \sigma_{d}^{0.7}\right)$ with an exponent of 0.7 for any range of the crystallinity can be deduced. For $\mu c-\mathrm{Si}: \mathrm{H}$ the data reported in Ref. 83 are in good agreement with this result, whereas lower value of the exponent $0.43-0.5$ were reported in Refs. 82, 84, and 85 . The relation obtained on the material with very low or no crystallinity presented in Fig. 4(c) is in very good agreement with data on $a-\mathrm{Si}: \mathrm{H}$ (Ref. 73) for the region of $\sigma_{d}<10^{-7} \mathrm{~S} / \mathrm{cm}$.

Two findings from Fig. 4 are important. First of all, the slope of the power-law dependence between $\sigma_{\mathrm{ph}}$ and $\sigma_{d}$ seems independent on the crystallinity. This suggests a common mechanism irrespective of the microstructure. The variation in the occupation of defects which act as recombination centers is such a mechanism. For $n$-type material, the double occupation of the majority of defect states leads to the reduction in the recombination probability and to an increase in the excess carrier lifetime. ${ }^{90}$ Second, $\sigma_{\mathrm{ph}}$ is similar at close values of $\sigma_{d}$ for samples with about ten times higher doping levels and accordingly higher defect densities. This clearly shows that unlike in intrinsic amorphous silicon, the total density of defects is of minor importance for $\sigma_{\mathrm{ph}}$ in doped amorphous and microcrystalline silicon. The occupation of the states in the gap is the most important parameter.

A key question is the spatial distribution of the defects. In a previous paragraph, an inhomogeneous distribution of defects is suggested with preferential defect location in the grain boundaries or in the amorphous phase for the interpretation of the results on intrinsic microcrystalline samples. This idea can also be used to explain the results obtained on the doped $\mu c-\mathrm{Si}: \mathrm{H}$. It is proposed that the Fermi energy is shifted toward midgap by the additional defects which are separated from the volume of the crystalline grains, e.g., in grain boundaries or the amorphous phase. As the recombination centers which control the recombination rate are located in the crystalline volume, their occupation will be changed and the photoconductivity will be changed as in the case of amorphous silicon. This means the occupation of the defects within the grains is controlled by the density of defects at the grain boundaries. The spatial separation of the photogenerated carriers from the additional defects could be supported by the buildup of a space-charge region caused by the charge transfer from the donors to the spatially separated defects. In this case, the minority carriers (holes) are captured at the defects and the lifetime of the spatially separated majority carriers increases.

One additional finding of Fig. 4 is noticeable. In the Raman amorphous samples [Fig. 4(c)] the photoconductivity changes by more than 1 order of magnitude whereas the dark conductivity, i.e., the Fermi energy, remains constant. Therefore, these samples can be considered as intrinsic after irradiation and the change in the density of neutral defects dominates the lifetime of the photogenerated carriers. After a few annealing steps, the shift of $E_{F}$ sets in and the photoconductivity changes in a common manner, i.e., is controlled by the Fermi level and no longer by the density of neutral defects.

\section{F. Use of photoconductivity spectroscopy in CPM mode for evaluation of defect density in thin-film silicon}

Optical subband gap absorption can be used for determination of the defect density in thin-film silicon materials and 
related alloys. The optical absorption as a function of the photon energy $E=h \nu$ is linked to the density of states through the relation $\alpha=$ const $h \nu \times R^{2}(h \nu) \times J(h \nu)$ where $R$ is the average dipole matrix element for the optical transitions and $J$ the joint density of states of initial and final state.

Popular methods to measure optical absorption on thinfilm silicon materials with high resolution are PDS (Ref. 91) and CPM. ${ }^{53}$ Both methods rely on a number of assumptions. For CPM we can summarize the main requirements as: ${ }^{52-54,92}$ (i) uniform illumination, i.e., thickness $(d) x$ absorption coefficient $(\alpha) \ll 1$, (ii) no changes in the transport path, which is of importance in a heterogeneous material such as $\mu c-\mathrm{Si}: \mathrm{H}$ or when the material is changed by electron bombardment, and (iii) no spectral dependence of carrier density and charge state of the recombination centers and a resulting constant mobility-lifetime product. $^{53}$

The application of both CPM and PDS for evaluation of the defect density is established for $a$-Si:H. Methods to identify the contribution from the defects on the absorption spectra have been investigated and described in detail in the literature. ${ }^{52,53,92-96}$ For $\mu c-\mathrm{Si}: \mathrm{H}$ the relation between defect absorption measured with PDS and spin density was recently investigated. ${ }^{62}$ It was seen that an almost linear relationship between the defect absorption and $N_{s}$ exists over 3 orders of magnitude, but there are also considerable deviations from this behavior which makes use of PDS for optimization difficult. For the application of CPM in $\mu c-\mathrm{Si}: \mathrm{H}$, convincing evidence that it can be used over a wide range of defect densities is missing so far.

From the data presented here, there are two main observations concerning the application of CPM in the entire range of TFS materials and over a wide range of defect densities: (i) deviation from a linear relationship $\alpha_{D} \propto N_{S}^{1.0}$ for $a-\mathrm{Si}: \mathrm{H}$ with very high defect density and (ii) complete absence of a quantitative relationship in $\mu c-\mathrm{Si}: \mathrm{H}$

\section{G. Amorphous silicon}

From the results shown in Fig. 2(a) we conclude that the CPM is a valid and useful method for the determination of a relative value of the optical absorption $\alpha$ and of the defect density in intrinsic $a-\mathrm{Si}: \mathrm{H}$ over at least 2 orders of magnitude $\left(N_{S}=10^{16}-10^{18} \mathrm{~cm}^{-3}\right)$. Use of the defect absorption at a specific energy (here $1.2 \mathrm{eV}$ ) yields good proportionality with the defect density measured with electron-spin resonance without need for a simulation with a model density of states.

The amorphous materials prepared with different SC resulting in possible different MRO do not show differences in the evaluated relationship $\alpha_{D}$ vs $N_{S}$. All show a good linear dependency up to $N_{S}=10^{18} \mathrm{~cm}^{-3}$ and nonlinear dependence at higher spin densities.

The results also support our assumption that the defect generation and annealing in powder and substrate samples behave similar so that we can use the spin density measured on powder for evaluation of conductivity vs defect density (Fig. 2).

The nonlinear dependence above $10^{18} \mathrm{~cm}^{-3}$ remains puzzling. The key problem for the explanation is finding a defect density-related mechanism for excitation or/and recombination of the carriers from the defect states which would be different from the mechanism for the carriers excited via band-to-band transition. For example, the recombination rate of the carriers excited via defect-band and band-to-band transitions is different and the difference changes as the defect density is varied. This should show up as a change in the carrier lifetime at least in the subgap region. It is monitored by measuring the phase shift of the CPM signal with respect to the excitation. In the case of $a-\mathrm{Si}: \mathrm{H}$ the phase of the signal was nearly the same over the whole spectrum, i.e., no significant variation in the recombination kinetics was observed. We must conclude that the CPM method needs a re-evaluation for the application in very high defect density $a-\mathrm{Si}: \mathrm{H}$ material, where some of the basic assumptions are not valid.

\section{H. Microcrystalline silicon}

For $\mu c-\mathrm{Si}: \mathrm{H}$ the evaluation of the defect density from the CPM in our experiment is only of qualitative value, i.e., the subgap absorption increases with irradiation and decreases with annealing. Any systematic and quantitative relationship is not seen.

We suggest two explanations. (i) The assumptions necessary for the use of CPM such as a constant $\eta \mu \tau$ product and no change in transport path are not valid in the heterogeneous material $\mu c-\mathrm{Si}: \mathrm{H}$ because the transport is heavily affected by the detailed interaction of carriers with the crystalline regions, the grain boundaries, and the amorphous phase. This interaction could be strongly affected by the irradiation and annealing experiment, which leads to the second point. (ii) The experimental procedure of irradiation and annealing of defects affects the $\mu c-\mathrm{Si}: \mathrm{H}$ in a special way, in particular differently in the various structure phases. We have already mentioned such a possibility above in our discussion of the variation of $\sigma_{\mathrm{ph}}$ in $\mu c-\mathrm{Si}: \mathrm{H}$ as a function of the defect density. In this respect, namely, application of CPM for the determination of the defect density, the experiment of electron bombardment, and annealing might not be the ideal tool for variation in the defect density in $\mu c-\mathrm{Si}: \mathrm{H}$. But we can also expect similar inhomogeneous defect generation to occur during the growth of $\mu c-\mathrm{Si}: \mathrm{H}$ material in general, resulting in similar effects on transport. We would therefore have to discourage from the use of the CPM for the evaluation of the defect density in $\mu c-\mathrm{Si}: \mathrm{H}$ for, e.g., material and growth optimization. Future studies should consider this carefully.

\section{CONCLUSIONS}

The influence of the defect density on charge carrier transport was investigated in thin-film silicon with structure composition all the way from highly crystalline to amorphous. The defect density is confirmed a most critical parameter for the performance of carrier transport which will be important for material and device optimization. An experiment where defects are generated by electron bombardment and then stepwise reduced by annealing is shown to be very useful for these investigations. 
(i) For intrinsic $a-\mathrm{Si}: \mathrm{H}$ a perfect agreement to the relationship $\sigma_{\mathrm{ph}} \propto N_{S}^{-1}$, in accordance with the recombination dominated by direct capture with neutral db's, is found over more than 3 orders of magnitude. Raman-amorphous material prepared with high hydrogen dilution behaves in all investigated aspects of carrier transport and recombination such as the pure amorphous material. (ii) For intrinsic $\mu c-\mathrm{Si}: \mathrm{H}$ the absence of the relationship $\sigma_{\mathrm{ph}} \propto N_{S}^{-1}$ is attributed to the heterogeneous structure with inhomogeneous distribution of defects in the amorphous phase, crystalline phase, and grain boundaries. (iii) In $n$-type phosphorusdoped material a universal influence of the Fermi-level shift controlled by doping and by the variation in the density of deep defects on the photoconductivity is found in all thinfilm silicon materials investigated here. It is concluded that the charge state of defects has the dominant influence on the photoconductivity via its influence on the carrier lifetime. (iv) The usefulness of the CPM spectroscopy for determination of the defect density is confirmed for $a-\mathrm{Si}: \mathrm{H}$ for a wide range of defect densities. However for $N_{S}>10^{18} \mathrm{~cm}^{-3}$, the
CPM underestimates the defect density as for such high densities the assumptions for CPM are no longer valid, which will have to be considered in future evaluations. (v) For $\mu c-\mathrm{Si}: \mathrm{H}$, the CPM fails to be an appropriate method for the measurement of defect density over any wider range of densities. An inhomogeneous distribution of defects in the heterogeneous structure has to be assumed which renders the assumptions for CPM invalid.

\section{ACKNOWLEDGMENTS}

We thank M. Hülsbeck, A. Lambertz, J. Klomfass (all IEF5-PV), and K. Petter (HMI Berlin) for their contributions to this work; I. Neklyudov (NSC KIPT) for the support of the electron bombardment experiments in Ukraine. This work is partially financially supported by the Bundesministerium für Bildung und Forschung in the project EPRSOLAR (Contract No. 03SF0328D) and European Community via Science and Technology Center in Ukraine (Project No. 655A).
${ }^{1}$ R. A. Street, Hydrogenated Amorphous Silicon (Cambridge University Press, New York, 1991).

${ }^{2}$ M. Stutzmann, W. B. Jackson, and C. C. Tsai, Phys. Rev. B 32, 23 (1985).

${ }^{3}$ A. R. Hepburn, J. M. Marshall, C. Main, M. J. Powell, and C. van Berkel, Phys. Rev. Lett. 56, 2215 (1986).

${ }^{4}$ R. Brüggemann and C. Main, Phys. Rev. B 57, R15080 (1998).

${ }^{5}$ K. Lips, P. Kanschat, and W. Fuhs, Sol. Energy Mater. Sol. Cells 78, 513 (2003)

${ }^{6}$ K. Lips, P. Kanshat, D. Will, C. Lerner, and W. Fuhs, J. NonCryst. Solids 227-230, 1021 (1998).

${ }^{7}$ S. Klein, F. Finger, R. Carius, and M. Stutzmann, J. Appl. Phys. 98, 024905 (2005).

${ }^{8}$ S. Klein, T. Repmann, and T. Brammer, Sol. Energy 77, 893 (2004).

${ }^{9}$ J. Müller, F. Finger, R. Carius, and H. Wagner, Phys. Rev. B 60, 11666 (1999).

${ }^{10}$ T. Dylla, Electron-Spin Resonance and Transient Photocurrent Measurements on Microcrystalline Silicon, in Schriften des Forschungszentrums Jülich, Energietechnik Vol. 43 (Forschungszentrum Jülich GmbH, Jülich, 2005).

${ }^{11}$ F. Finger, L. B. Neto, R. Carius, T. Dylla, and S. Klein, Phys. Status Solidi C 1, 1248 (2004)

${ }^{12}$ M. Kondo, S. Yamasaki, and A. Matsuda, J. Non-Cryst. Solids 266-229, 544 (2000).

${ }^{13}$ M. M. de Lima, P. C. Taylor, S. Morrison, A. LeGeune, and F. C. Marques, Phys. Rev. B 65, 235324 (2002).

${ }^{14}$ K. Morigaki, H. Hikita, M. Yamaguchi, and F. Fujita, Mater. Sci. Eng., B 103, 37 (2003).

${ }^{15}$ O. Astakhov, R. Carius, A. Lambertz, Yu. Petrusenko, V. Borysenko, D. Barankov, and F. Finger, J. Non-Cryst. Solids 354, 2329 (2008).

${ }^{16}$ C. Longeaud, J. P. Kleider, P. Roca i Cabarrocas, S. Hamma, R. Meaudre, and M. Meaudre, J. Non-Cryst. Solids 227-230, 96 (1998).

${ }^{17}$ C. R. Wronski, J. M. Pearce, R. J. Koval, X. Niu, A. S. Ferlauto, J. Koh, and R. W. Collins, Mater. Res. Soc. Symp. Proc. 715, A13.4 (2002).
${ }^{18}$ X. Xu, J. Yang, and S. Guha, Appl. Phys. Lett. 62, 1399 (1993).

${ }^{19}$ S. Guha, J. Yang, A. Banerjee, Y. Baojie, and K. Lord, Sol. Energy Mater. Sol. Cells 78, 329 (2003).

${ }^{20}$ S. M. Nakhmanson, P. M. Voyles, N. Mousseau, G. T. Barkema, and D. A. Drabold, Phys. Rev. B 63, 235207 (2001).

${ }^{21}$ J. P. Kleider, C. Longeaud, M. Gauthier, M. Meaudre, R. Meaudre, R. Butte, S. Vignoli, and P. Roca i Cabarrocas, Appl. Phys. Lett. 75, 3351 (1999).

${ }^{22}$ P. Roca i Cabarrocas, A. Fontcuberta i Morral, and Y. Poissant, Thin Solid Films 403-404, 39 (2002).

${ }^{23}$ P. St'ahel, S. Hamma, P. Sladek, and P. Roca i Cabarrocas, J. Non-Cryst. Solids 227-230, 276 (1998).

${ }^{24}$ S. Guha, J. Yang, D. L. Williamson, Y. Lubianiker, J. D. Cohen, and A. H. Mahan, Appl. Phys. Lett. 74, 1860 (1999).

${ }^{25}$ A. L. Baia Neto, A. Lambertz, R. Carius, and F. Finger, Phys. Status Solidi A 186, R4 (2001).

${ }^{26}$ D. V. Tsu, B. S. Chao, and S. J. Jones, Sol. Energy Mater. Sol. Cells 78, 115 (2003).

${ }^{27}$ S. Guha, Mater. Res. Soc. Symp. Proc. 808, A6.4.1 (2004).

${ }^{28}$ O. Astakhov, F. Finger, R. Carius, A. Lambertz, Yu. Petrusenko, V. Borysenko, and D. Barankov, J. Non-Cryst. Solids 352, 1020 (2006).

${ }^{29}$ O. Astakhov, R. Carius, Yu. Petrusenko, V. Borysenko, D. Barankov, and F. Finger, Phys. Status Solidi (RRL) 1, R77 (2007).

${ }^{30}$ O. Astakhov, R. Carius, Yu. Petrusenko, V. Borysenko, D. Barankov, and F. Finger, Mater. Res. Soc. Symp. Proc. 989, A02 (2007).

${ }^{31}$ O. Astakhov, F. Finger, R. Carius, A. Lambertz, Yu. Petrusenko, V. Borysenko, and D. Barankov, Thin Solid Films 515, 7513 (2007).

${ }^{32}$ R. Street, D. Biegelsen, and J. Stuke, Philos. Mag. B 40, 451 (1979).

${ }^{33}$ U. Voget-Grote, W. Kummerle, R. Fischer, and J. Stuke, Philos. Mag. B 41, 127 (1980).

${ }^{34}$ W. M. Pontuschka, W. W. Carlos, P. C. Taylor, and R. W. Griffith, Phys. Rev. B 25, 4362 (1982).

${ }^{35}$ H. Dersch, L. Schweitzer, and J. Stuke, Phys. Rev. B 28, 4678 
(1983).

${ }^{36}$ H. Dersch, A. Skumanich, and N. M. Amer, Phys. Rev. B 31, 6913 (1985).

${ }^{37}$ F. Finger, W. Fuhs, and R. Carius, Philos. Mag. Lett. 57, 235 (1988).

${ }^{38}$ R. Könenkamp and E. Wild, Phys. Rev. B 42, 5887 (1990).

${ }^{39}$ P. Danesh, B. Pantchev, and E. Vlaikova, Nucl. Instrum. Methods Phys. Res. B 239, 370 (2005).

${ }^{40}$ R. Carius and F. Finger, J. Non-Cryst. Solids 114, 549 (1989).

${ }^{41}$ H. Schade and J. I. Pankove, J. Phys. Colloq. C4, 327 (1981).

${ }^{42}$ U. Schneider and B. Schröder, Solid State Commun. 69, 895 (1989).

${ }^{43}$ U. Schneider, A. Scholz, B. Schröder, F. Karg, and H. Kausche, Jpn. J. Appl. Phys., Part 1 30, 228 (1991).

${ }^{44}$ F. Diehl, W. Herbst, S. Bauer, B. Schröder, and H. Oechsner, J. Non-Cryst. Solids 198-200, 436 (1996).

${ }^{45}$ A. Yelon, H. Fritzsche, and H. M. Branz, J. Non-Cryst. Solids 266-269, 437 (2000).

${ }^{46}$ T. Shimizu, M. Iwami, T. Okagawa, A. Morimoto, and M. Kumeda, Mater. Res. Soc. Symp. Proc. 258, 455 (1992).

${ }^{47}$ C. Malten, F. Finger, P. Hapke, T. Kulessa, C. Walker, R. Carius, R. Flückiger, and H. Wagner, Mater. Res. Soc. Symp. Proc. 358, 757 (1995)

${ }^{48}$ R. Brüggemann, W. Bronner, and M. Mehring, Solid State Commun. 119, 23 (2001)

${ }^{49}$ R. Brüggemann, J. P. Kleider, W. Bronner, and I. Zrinscak, J. Non-Cryst. Solids 299-302, 632 (2002).

${ }^{50}$ W. Bronner, M. Mehring, and R. Brüggemann, Phys. Rev. B 65 , 165212 (2002).

${ }^{51}$ M. Stutzmann and D. K. Biegelsen, Phys. Rev. B 40, 9834 (1989).

${ }^{52}$ J. Kočka, M. Vaněček, and A. Tříska, in Advances in Disordered Semiconductors, edited by H. Fritzsche (World Scientific, Singapore, 1989), Vol. 1, p. 297.

${ }^{53}$ M. Vaněček, J. Kočka, J. Stuchlik, and A. Tříska, Solid State Commun. 39, 1199 (1981).

${ }^{54}$ K. Pierz, W. Fuhs, and H. Mell, Philos. Mag. B 63, 123 (1991).

${ }^{55}$ H. Stiebig and F. Siebke, Philos. Mag. B 72, 489 (1995).

${ }^{56}$ P. V. Santos, W. B. Jackson, and R. A. Street, Phys. Rev. B 44, 12800 (1991).

${ }^{57}$ R. Krankenhagen, M. Schmidt, W. Henrion, I. Sieber, S. Koynov, S. Grebner, and R. Schwarz, Diffus. Defect Data, Pt. B 47, 607 (1996)

${ }^{58}$ M. Vaněček, A. Poruba, Z. Remeš, J. Rosa, S. Kamba, V. Vorlíček, J. Meier, and A. Shah, J. Non-Cryst. Solids 266-260, 519 (2000).

${ }^{59}$ M. Güneş, D. Akdaş, O. Göktaş, R. Carius, J. Klomfass, and F. Finger, J. Mater. Sci. 14, 729 (2003).

${ }^{60}$ A. L. Baia Neto, A. Lambertz, R. Carius, and F. Finger, J. NonCryst. Solids 299-302, 274 (2002).

${ }^{61}$ M. Vanecek and A. Poruba, Appl. Phys. Lett. 80, 719 (2002).

${ }^{62}$ S. Klein, F. Finger, R. Carius, T. Dylla, and J. Klomfass, J. Appl. Phys. 102, 103501 (2007)

${ }^{63}$ L. Houben, M. Luysberg, P. Hapke, R. Carius, F. Finger, and H. Wagner, Philos. Mag. A 77, 1447 (1998).

${ }^{64}$ K. Awazu, K. Watanabe, and H. Kawazoe, J. Appl. Phys. 73, 8519 (1993).

${ }^{65}$ C. Malten, Pulsed Electron-Spin Resonance of Amorphous and Microcrystalline Semiconductors, in Berichte des Forschungszentrum Jülich Vol. 3273 (RWTH Aachen, Forschungszentrum,
Jülich 1996).

${ }^{66}$ K. Petter, O. Astakhov, K. Lips, and F. Finger (unpublished).

${ }^{67}$ C. Smit, R. A. C. M. M. van Swaaij, H. Donker, A. M. H. N. Petit, W. M. M. Kessels, and M. C. M. van de Sanden, J. Appl Phys. 94, 3582 (2003).

${ }^{68}$ A. L. Baia Neto, T. Dylla, S. Klein, T. Repmann, A. Lambertz, R. Carius, and F. Finger, J. Non-Cryst. Solids 338-340, 168 (2004).

${ }^{69}$ W. E. Spear, G. Willeke, and P. G. LeComber, Physica B \& C 117-118, 908 (1983).

${ }^{70}$ U. Backhausen, R. Carius, F. Finger, P. Hapke, U. Zastrow, and H. Wagner, Mater. Res. Soc. Symp. Proc. 452, 833 (1997).

${ }^{71}$ F. Finger, J. Müller, C. Malten, R. Carius, and H. Wagner, J. Non-Cryst. Solids 266-269, 511 (2000).

${ }^{72}$ T. Dylla, R. Carius, and F. Finger, Mater. Res. Soc. Symp. Proc. 715, A20.9.1 (2002).

${ }^{73}$ W. Beyer and B. Hoheisel, Solid State Commun. 47, 573 (1983).

${ }^{74}$ P. E. Vanier, Sol. Cells 9, 85 (1983).

${ }^{75}$ J. Stuke, J. Non-Cryst. Solids 97-98, 1 (1987).

${ }^{76}$ P. Pipoz, E. Sauvain, J. Hubin, and A. Shah, Mater. Res. Soc. Symp. Proc. 258, 777 (1992).

${ }^{77}$ N. Beck, N. Wyrsch, Ch. Hof, and A. Shah, J. Appl. Phys. 79, 9361 (1996).

${ }^{78} \mathrm{H}$. Overhof and P. Thomas, Electronic Transport in Hydrogenated Amorphous Semiconductors (Springer-Verlag, New York, 1989).

${ }^{79}$ J. W. Corbett, Electron Radiation Damage in Semiconductors and Metals (Academic, New York, 1966).

${ }^{80}$ S. Klein, F. Finger, R. Carius, and J. Lossen, Thin Solid Films 501, 43 (2006).

${ }^{81}$ S. K. Ram, S. Kumar, and P. Roca i Cabarrocas, Thin Solid Films 515, 7469 (2007).

${ }^{82}$ F. Finger, S. Klein, R. Carius, T. Dylla, O. Vetterl, and A. L. Baia Neto, J. Mater. Sci. 14, 621 (2003).

${ }^{83}$ V. Smirnov, S. Reynolds, C. Main, F. Finger, and R. Carius, J. Non-Cryst. Solids 338-340, 421 (2004).

${ }^{84}$ R. Brüggemann and N. Souffi, J. Non-Cryst. Solids 352, 1079 (2006).

${ }^{85}$ N. Souffi, G. H. Bauer, and R. Brüggemann, Thin Solid Films 501, 129 (2006).

${ }^{86}$ J. Müller, Electron-Spin Resonance Studies on Microcrystalline Silicon, in Berichte des Forschungszentrums Jülich Vol. 3615 (Forschungszentrum Jülich GmbH, Jülich, 1998).

${ }^{87}$ T. Bronger and R. Carius, Thin Solid Films 515, 7486 (2007).

${ }^{88}$ T. Merdzhanova, R. Carius, S. Klein, F. Finger, and D. DimovaMalinovska, Thin Solid Films 511-512, 394 (2006).

${ }^{89}$ T. Dylla, S. Reynolds, R. Carius, and F. Finger, J. Non-Cryst. Solids 352, 1093 (2006).

${ }^{90}$ D. A. Anderson and W. E. Spear, Philos. Mag. 36, 695 (1977).

${ }^{91}$ W. B. Jackson and N. M. Amer, Phys. Rev. B 25, 5559 (1982).

${ }^{92}$ E. Sauvain, A. Mettler, N. Wyrsch, and A. Shah, Solid State Commun. 85, 219 (1993).

${ }^{93}$ N. Wyrsch, F. Finger, T. J. McMahon, and M. Vanecek, J. NonCryst. Solids 137-138, 347 (1991).

${ }^{94}$ F. Siebke, H. Stiebig, A. Abo-Arais, and H. Wagner, Sol. Energy Mater. Sol. Cells 41-42, 529 (1996).

${ }^{95}$ J. A. Schmidt and F. A. Rubinelli, J. Appl. Phys. 83, 339 (1998).

${ }^{96}$ C. Main, S. Reynolds, I. Zrinscak, and A. Merazga, J. NonCryst. Solids 338-340, 228 (2004). 